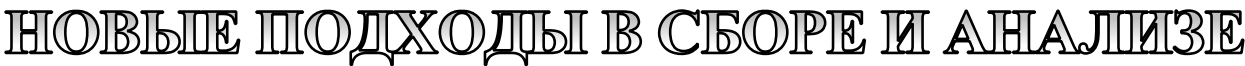

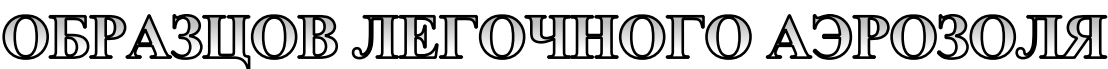

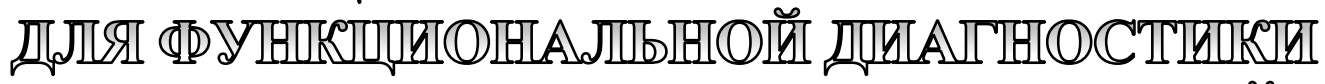

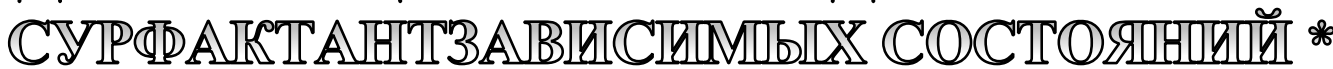

\author{
А.И. Мизев, Институт механики сплошных сред УрО РАН \\ А.В. Шмыров, Институт механики сплошных сред УрО РАН \\ И.А. Мизева, Институт механики сплошных сред УрО РАН \\ А.И. Шмырова, Институт механики сплошных сред УрО РАН \\ И.М. Пшеничникова-Пеленева, Пермский государственный медицинский \\ университет им. академика Е.А. Вагнера
}

\section{Для цитирования:}

Мизев А.И., Шмыров А.В., Мизева И.А., Шмырова А.И., Пшеничникова-Пеленева И.М. Новые подходы в сборе и анализе образцов легочного аэрозоля для функциональной диагностики сурфактантзависимых состояний // Вестник Пермского федерального исследовательского центра. - 2021. - № 1. C. 64-72. https://doi.org/10.7242/2658-705X/2021.1.6

Разработка эфффективных методов неинвазивного сбора образцов легочного аэрозоля и изучения поверхностной активности полученного нативного материала актуальна для построения диагностики воспалительных пневмопатий легких. В результате исследования разработан и аппаратно реализован метод, основанный на улавливании капель легочной жидкости, содержащихся в виде аэрозоля в выдыхаемом человеком воздухе. Поток выдыхаемого воздуха пропускается через область коронного разряда, где капли аэрозоля получают электрический заряд и затем переносятся на поверхность воды электростатическими силами. Содержащийся в захваченных каплях легочный сурфактант формирует адсорбированный слой, поверхностные свойства которого затем исследуются тензиометрическими методами.

Апробация метода и оптимизация конструкции установки проведены на модельном аэрозоле слабого раствора электролита, что позволило измерять эфффективность захвата через проведение кондуктометрических измерений. Для исследования поверхностных свойств легочного сурфактанта использован модифицированный метод капиллярных волн. Методика сбора выдыхаемых частиц и исследования поверхностных свойств легочного сурфактанта протестирована на группе здоровых добровольцев. Проведено сравнительное исследование поверхностных свойств нативного материала, собранного электростатическим методом из

\footnotetext{
* Работа выполнена при финансовой поддержке РФФИ № (проект № 17-41-590095 p-а).
} 
выдыхаемого воздуха и полученного в процессе бронхоальвеолярного смыва в группах здоровых добровольцев и больных туберкулезом легких.

Ключевые слова: легочный сурфактант, поверхностное натяжение, капиллярные волны, туберкулез.

\section{Введение}

Дыхание человека сопровождается формированием капелек легочной жидкости, которые в виде аэрозоля поступают в окружающую среду с выдыхаемым воздухом. Механизм формирования капелек связан с открытием и закрытием мелких дыхательных путей во время дыхания [1-3]. Выдыхаемый воздух здорового человека во время нормального дыхания содержит в среднем несколько субмикронных частиц в $1 \mathrm{~cm}^{3}$ [4-6], состав которых идентичен составу легочной жидкости [7-9]. Размер и концентрация выдыхаемых частиц могут значительно варьироваться у разных людей [10] и зависят от типа дыхания $[11,12]$. Известно, что состав и свойства легочной жидкости могут существенно различаться у здоровых и пациентов с различными легочными заболеваниями [13]. Таким образом, сбор выдыхаемого аэрозоля для последующего анализа состава с целью построения диагностической методики и контроля качества проводимого лечения является актуальной и важной задачей. Отметим, что на сегодняшний день самым надежным способом сбора образцов легочной жидкости является инвазивная и болезненная процедура взятия бронхоальвеолярного смыва (БАС) при проведении фибробронхоскопии [14-18]. Как правило, в клинических образцах состояние легочного сурфактанта оценивается путем изучения его поверхностной активности и биохимического состава. Высокое содержание легочного сурфактанта в образцах жидкости бронхоальвеолярного смыва дает возможность прямого использования методов тензиометрии для получения зависимости поверхностного давления от площади субфазы (изотерма Ленгмюра), необходимой для оценки индекса активности сурфактанта [14] и его сжимаемости [15].
Этот метод является инвазивным, трудоемким, имеет ряд противопоказаний. В качестве неинвазивного метода предлагается сбор конденсата выдыхаемого воздуха [19], однако этот метод отличается низкой эффективностью и воспроизводимостью результатов [20].

Методы электростатического захвата капелек достаточно эффективны [21] и используются для очистки воздуха и захвата капелек аэрозоля из выдыхаемого воздуха [22, 23].

Основной целью настоящего исследования является получение неинвазивным способом образцов легочного сурфактанта из выдыхаемого воздуха, который представляет собой поверхностно-активный комплекс липопротеинов [24]. Важной ролью легочного сурфактанта является понижение поверхностного натяжения альвеолярной жидкости, что позволяет легче осуществлять вдох. Поверхностноактивные свойства легочного сурфактанта обусловлены количеством, соотношением и взаимодействием входящих в его состав фосфолипидов, формирующих монослойную мембрану на разделе фаз жидкостьвоздух. Уникальной способностью слоя легочного сурфактанта является понижение поверхностного натяжения до очень низких значений, что предотвращает схлопывание альвеол в конце выдоха. Изменения в молекулярной структуре легочного сурфактанта могут значительно снизить его поверхностную активность и, как следствие, привести к развитию дисфункции легочного сурфактанта [25].

Различные заболевания (бронхиальная астма и ХОБЛ, диффузный пневмофиброз, диссеминированные процессы в легких различного генеза, бактериальная, вирусная, грибковая пневмония, ОРДС, туберкулез легких, некоторые экстраторакальные заболевания) приводят к измене- 
нию состава легочного сурфактанта, снижают его поверхностную активность, что провоцирует альвеолярную нестабильностью и развитие дистелектазов, отека, воспаления и фиброзирования легочного интерстиция, нарушение проницаемости аэрогематического барьера и дыхательной недостаточности [26-34].

Раннее обнаружение нарушений функции легочного сурфактанта и его биохимического состава чрезвычайно важно при обследовании недоношенных детей с респираторным дистресс-синдромом новорожденных, ОРДС взрослых при шоках и полиорганной недостаточности, хронической обструктивной болезни легких, альвеолитах, прочих диссеминированных процессах в легких и других пневмопатиях с тяжелыми нарушениями дыхания, при определении показаний для заместительной терапии экзогенными сурфактантами с оценкой ее эффективности.

Таким образом, актуально решение задачи неинвазивного сбора выдыхаемого аэрозоля и разработка эффективного метода изучения активности легочного сурфактанта, которые позволят значительно улучшить функциональную диагностику многих заболеваний легких, а также найти новые подходы к их патогенетической терапии.

\section{Метод сбора образцов легочного сурфактанта}

Для неинвазивного сбора образцов легочного сурфактанта реализован метод электростатического улавливания заряженных капель аэрозоля альвеолярной жидкости (ESAT - electrostatic aerosol trapping system), содержащей легочный сурфактант (рис. 1). Выдыхаемый воздух от пациента 1 через стеклянную трубку 2 попадает в резервуар 3. После окончания выдоха клапан 4 переходит закрытое положение, а воздух из резервуара 3 с постоянным расходом поступает в улавливающую систему. Улавливающая система состоит из воздуховода и кюветы с водой, исполняющей роль мишени. На конце среза воздуховода установлена тонкая игла, подсоединенная к высоковольтному источнику.

Частицы аэрозоля, проходя через область коронного разряда, приобретают электрический заряд. Для улавливания заряженных капель в дне кюветы расположен заземленный электрод в виде тонкой трубки. Заряженные капельки аэрозоля, двигаясь под действием электростатических сил ко второму электроду, сталкиваются с поверхностью воды, формируя со временем адсорбированный слой на ее поверхности. Эффективность сбора, выражаемая как отношение числа частиц, осажденных на поверхность жидкости, к общему числу частиц, прошедших с выдыхаемым воздухом через трубку, зависит от величины поля и геометрических размеров системы улавливания.

Для оптимизации конструкции и поиска оптимальных параметров системы установка тщательно тестировалась на стенде, имитирующем процесс выдоха

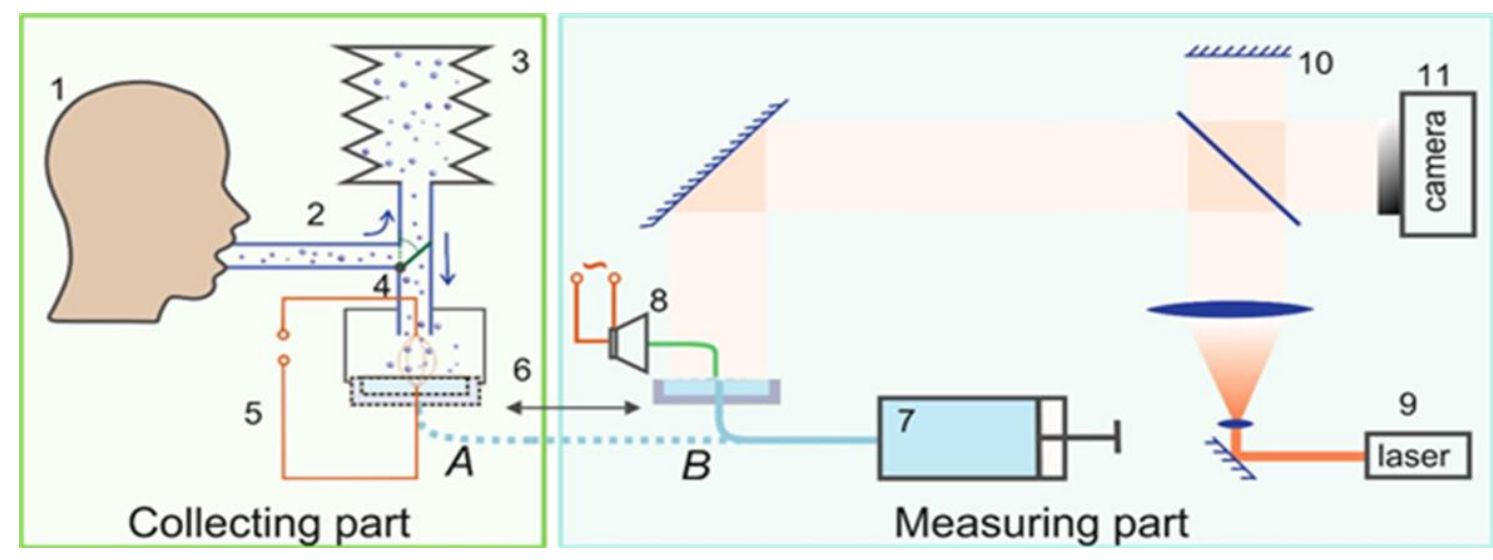

Рис. 1. Экспериментальная установка для электростатического захвата и осаждения на поверхности жидкости 
человека. В качестве жидкости для создания модельного аэрозоля использовался слабый раствор электролита (хлорида натрия), генерируемый небулайзером.

После окончания сбора нативного материала кювета 6 перемещается в измерительный модуль, состоящий из генератора звуковых колебаний и волновода 8 , которые передают волну давления на поверхность жидкости. Регистрация профиля поверхности производится при помощи интерферометрии с последующей расшифровкой интерферограммы. Детально особенности экспериментальной установки описаны в работе [35].

\section{Модифицированный метод капиллярных волн}

Для исследования физических свойств адсорбированной пленки, в частности изучения поверхностного натяжения и реологических характеристик, был модифицирован известный метод капиллярных волн. Новизной созданного метода является способ возбуждения и регистрации волны. Схема блока анализа собранного нативного материала представлена справа на рис. 1. Для бесконтактного возбуждения волны применено локальное акустическое воздействие. Детально установка описана в работах [36-37]. Использование цифровой интерферометрии и авторского алгоритма обработки данных позволяет проводить восстановление мгновенного профиля поверхности с точностью до 2 нм. Полученные в эксперименте изображения поверхности содержат крупномасштабные (колебание поверхности как целого) и мелкомасштабные (шум измерительной матрицы) шумы, кроме того, изображение включает в себя фрагменты измерительной установки, которые должны быть исключены из изображения. Характерный пример получаемого профиля волны показан на рис. 2. Эти особенности накладывают дополнительные ограничения на методы анализа профилей поверхности.

Реализовано два алгоритма обработки полученных профилей поверхности. В

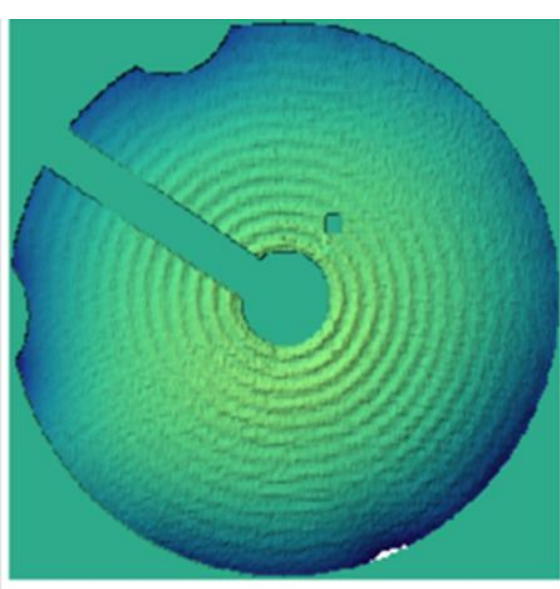

Рис. 2. Характерный профиль поверхности капиллярной волны, полученный на чистой воде при частоте возбуждения 2020 Ги

первом случае оператор в ручном режиме, используя полиномы Цернике вплоть до шестого порядка, вычитает крупномасштабные деформации поверхности раздела и далее полученный волновой профиль аппроксимируется уравнением затухающей цилиндрической волны. Целью построения второго алгоритма является быстрая автоматическая обработка сигнала. В его основе лежит разложение сигнала по уединенным цилиндрическим волнам различного масштаба. В целом алгоритм наследует основные принципы вейвлет-разложения, но, строго говоря, не является таковым. Оба алгоритма позволяют с достаточной точностью определить как длину капиллярной волны, необходимой для расчета поверхностного давления (П), так и коэффициент ее затухания $(\beta)$, отвечающий за реологические свойства формирующихся пленок поверхностно-активных веществ.

\section{Результаты исследования легочного} сурфактанта здоровых добровольцев и больных туберкулезом

Методика сбора выдыхаемых частиц и исследования поверхностных свойств собранного нативного материала была отработана на группе здоровых добровольцев (10 человек в возрасте от 25 до 70 лет разного пола со взятием от 1 до 5 проб). На рис. 3 представлены типичные зависимости поверхностного давления (П) 

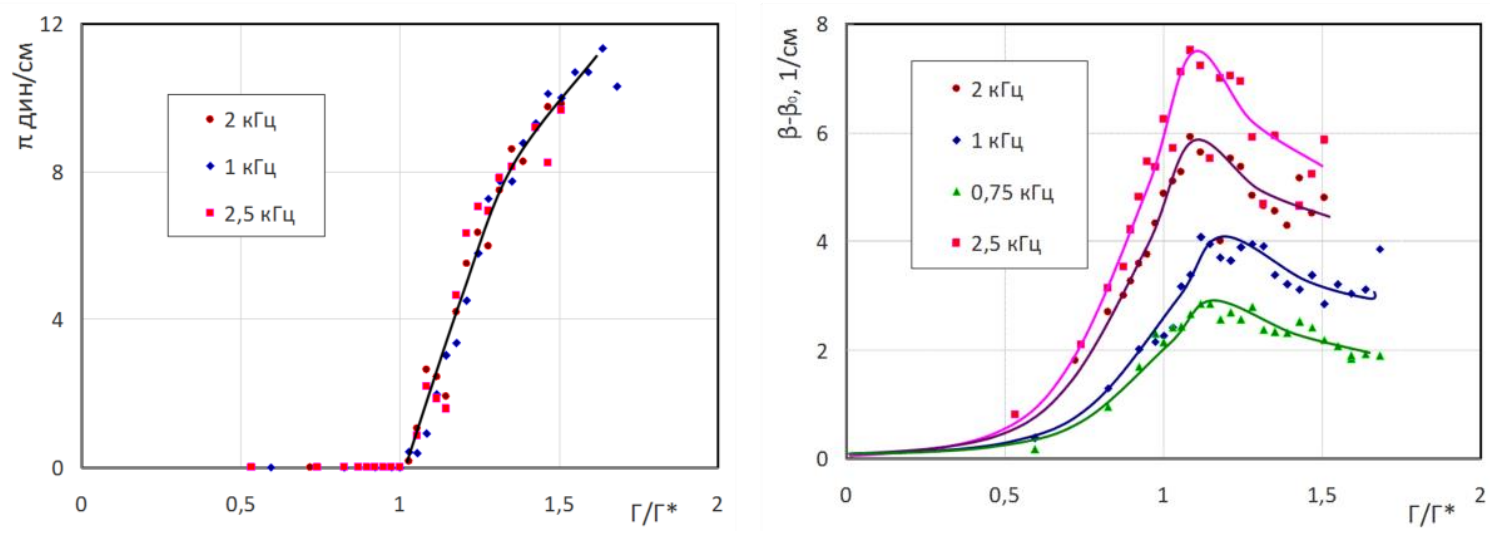

Рис. 3. Типичная зависимость поверхностного давления (П) и коэффициента затухания волны ( $\beta$ ) от безразмерной поверхностной кониентрации сурфактанта (Г) для различных частот возбуждения капиллярной волнь

и коэффициента затухания капиллярной волны ( $\beta)$ от безразмерной поверхностной концентрации сурфактанта $\left(\Gamma / \Gamma^{*}\right)$. При построении данной зависимости было использовано нормирование на поверхностную концентрацию сурфактанта, соответствующую концентрации фазового перехода поверхностной фазы $\Gamma^{*}$.

У двух здоровых добровольцев был проведен неинвазивный сбор проб легочного сурфактанта методом ESAT, и для сопоставления у двух добровольцев были собраны биопробы методом БАС при фибробронхосокопии (рис. 4). Для этого во втором случае адсорбированный слой создавался нанесением небольшого, порядка 30 мкл, количества смыва на поверхность чистой воды в измерительной ячейке. Обнаружено расхождение изотерм, полученных для этих двух нативных материалов.

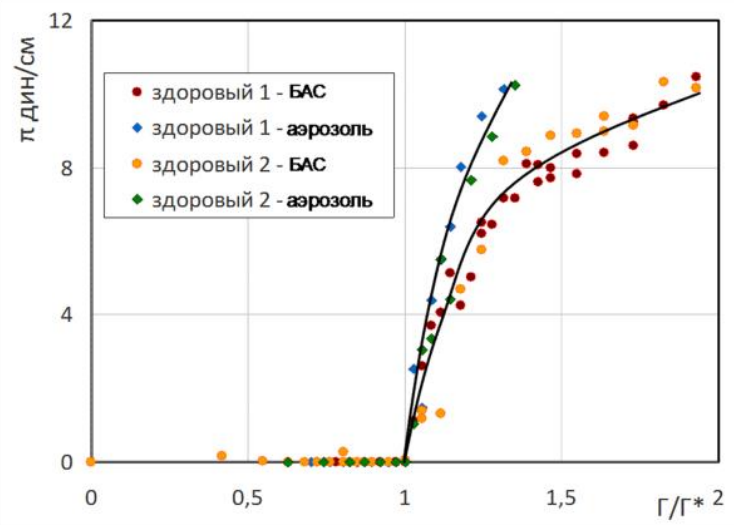

Возможным объяснением такого различия является разница в составе образцов, полученных разными методами, что обусловлено сбором материала из разных областей легкого. Из-за относительно большого диаметра зонда (несколько миллиметров) бронхоальвеолярный смыв делается как из бронхиальной, так и из альвеолярной части легкого. Причем большая часть смыва приходится на бронхиальную часть. В то же время механизм формирования выдыхаемых капелек аэрозоля таков, что они могут зарождаться только в самых дальних, наиболее близких к альвеолам, дыхательных путях, диаметр которых составляет несколько сотен микрометров. Из литературы известно, что составы альвеолярной и бронхиальной жидкости отличаются, что и отражается на их поверхностных свойствах

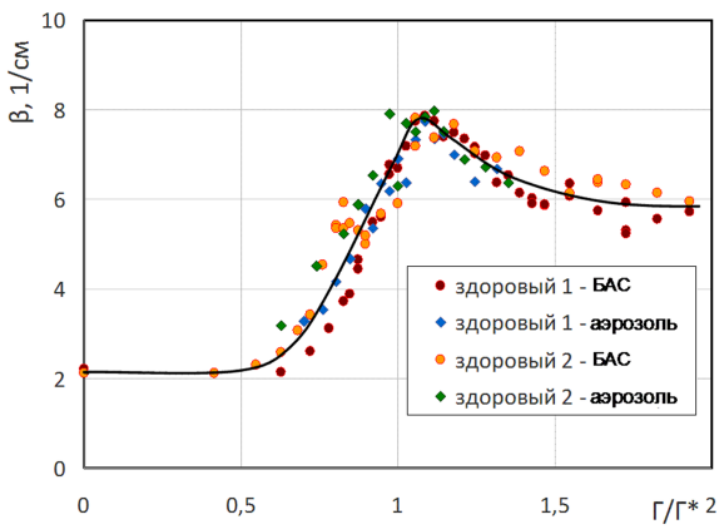

Рис. 4. Зависимости поверхностного давления и коэффициента затухания капиллярной волны от поверхностной концентрации материала, полученного путем сбора аэрозоля выдыхаемого воздуха и бронхоальвеолярного смыва у здоровых добровольиев 
Для клинического обоснования использования нового способа оценки поверхностно-активных свойств легочного сурфактанта были обследованы добровольцы, среди них 5 здоровых и 10 с воспалительными пневмопатиями (у пяти был выявлен туберкулез; четверых инфильтративный туберкулез с распространенностью менее одной доли легкого, у одного - диссеминированным туберкулез с распространенным двусторонним поражением). У всех больных образцы легочной жидкости были собраны инвазивным методом БАС и неинвазивным методом ESAT. Поверхностная активность изучалась модифицированным методом капиллярных волн, результаты представлены на рис. 5. Показано существенное отличие изотерм здоровых добровольцев и пациента с диссеминированной формой заболевания. В то же время изотермы, полученные у пациентов с ограниченным специфическим процессом, слабо отличаются от изотерм здоровой группы

\section{Выводы}

Изготовлен мобильный прототип устройства для сбора легочного сурфактанта и проведения исследований его поверхностных свойств в клинических условиях. Осаждение нативного материала на поверхность жидкости в виде адсорбированного слоя впервые дало возможность исследовать поверхностные свойства легочного сурфактанта, собранного неинвазивным

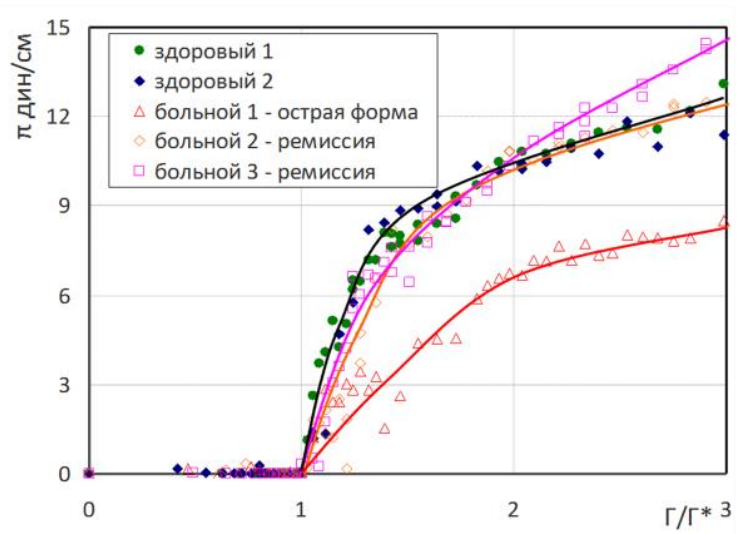

путем. До сих пор такие исследования были доступны только на базе материала, полученного инвазивными методами.

Важной частью работы является развитие тензиометрического метода [36] при этом существенно расширен доступный диапазон частот в высокочастотную область, где данному методу нет альтернативы среди других динамических методов исследования поверхностных и реологических свойств. Кардинальное уменьшение необходимого объема жидкости $(0,1$ мл против 10 мл) позволяет использовать метод в различных биохимических и биомедицинских приложениях.

Описанным модифицированным методом капиллярных волн изучена поверхностная активность образцов легочной жидкости, полученной двумя способами: инвазивным (БАС) и неинвазивным (ESAT). Показано расхождение поверхностной активности проб, полученных двумя методами, что объясняется различными источниками этих образцов, является интересным результатом и требует дальнейших исследований. Продемонстрировано существенное отличие изотерм нативного материала, полученного у здоровых добровольцев и пациента с диссеминированной формой заболевания. В то же время изотермы у пациентов с ограниченным специфическим процессом слабо отличаются от изотерм здоровой группы. Дальнейшее увеличе-

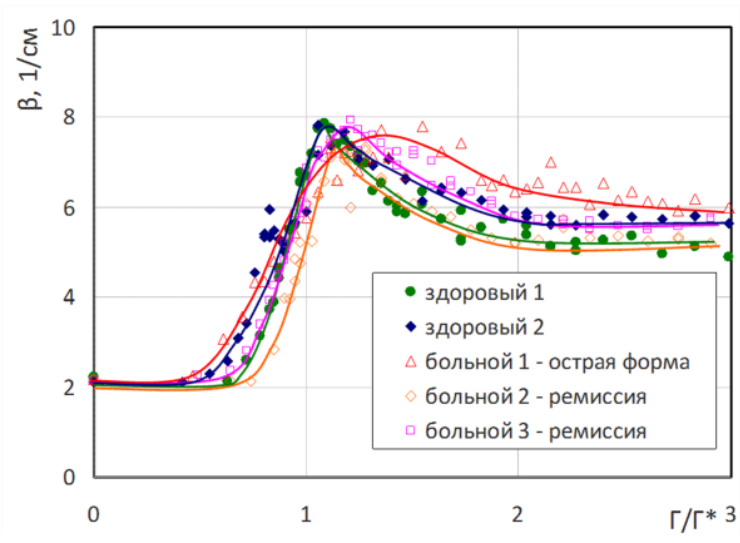

Рис. 5. Зависимости поверхностного давления и коэффициента затухания капиллярной волнь от поверхностной концентрации материала, полученного методом бронхоальвеолярного смыва у здоровых добровольиев и больных туберкулезом 
ние выборки групп сравнения позволит сделать вывод о клинической значимо- сти предложенной методики и возможности внедрения ее в практику.

\section{Библиографический список}

1. Haslbeck K., Schwarz K., Hohlfeld J.M., Seume J.R., Koch W. Submicron droplet formation in the human lung// Journal of Aerosol Science. - 2010. - Vol. 41. - P. 429-438.

2. Johnson G.R., Morawska L. The Mechanism of Breath Aerosol Formation // Journal of Aerosol Medicine and Pulmonary Drug Delivery. - 2009. - Vol. 22. - P. 229-237.

3. Almstrand A.C., Bake B., Ljungstrom E., Larsson P., Bredberg A., Mirgorodskaya E., Olin A.C. Effect of airway opening on production of exhaled particles // Journal of applied physiology. - 2010. - Vol. 108. - P. 584-588.

4. Fairchild C., Stampfer J. Particle concentration in exhaled breath. // American Industrial Hygiene Association Journal. - 1987. - Vol. 48. - P. 948-949.

5. Papineni R., Rosenthal F. The size distribution of droplets in the exhaled breath of healthy human subjects. Journal of Aerosol Medicine: Deposition, Clearance, and Effects in the Lung. - 1997. - Vol. 10. - P. 105-116.

6. Morawska L., Johnson G., Ristovski Z., Hargreaves M., Mengersen K., Corbett S., Chao C., Li Y., Katoshevski D. Size distribution and sites of origin of droplets expelled from the human respiratory tract during expiratory activities // Journal of Aerosol Science. - 2009. - Vol. 40. - P. 256-269.

7. Olin A.C. Particles in exhaled air - a novel method of sampling non-volatiles in exhaled air. // Volatile biomarkers: Non-invasive diagnosis in physiology and medicine Elsevier. - 2013. - Vol. 20. - P. 383-391.

8. Tinglev A.D., Ullah S., Ljungkvist G., Viklund E., Olin A.C., Beck O. Characterization of exhaled breath particles collected by an electret lter technique // Journal of Breath Research. - 2016. - Vol. 10. - P. 026001.

9. Ullah S., Sandqvist S., Beck O. Measurement of Lung Phosphatidylcholines in Exhaled Breath Particles by a Convenient Collection Procedure // Analitical Chemistry. - 2015. - Vol. 87. - P. 11553-11560.

10. Schwarz K., Biller H., Windt H., Koch W., Hohlfeld J.M. Characterization of exhaled particles from the healthy human lung|a systematic analysis in relation to pulmonary function variables. // Journal of aerosol medicine and pulmonary drug delivery. - 2010. - Vol. 23. P. 371-379.

11. Johnson G., Morawska L., Ristovski Z., Hargreaves M., Mengersen K., Chao C., Wan M., Li Y., Xie X., Katoshevski D. [et al] Modality of human expired aerosol size distributions. // Journal of Aerosol Science. 2011. - Vol. 42. - P. 839-851.

12. Bake B., Ljungstrom E., Claesson A., Carlsen H.K., Holm M., Olin A.C. Exhaled particles after a standardized breathing maneuver // Journal of aerosol medicine and pulmonary drug delivery. - 2017. Vol. 30. - P. 267-273.

13. Schwarz K., Biller H., Windt H., Koch W., Hohlfeld J.M. Characterization of exhaled particles from the human lungs in airway obstruction // Journal of aerosol medicine and pulmonary drug delivery. - 2015. Vol. 28. - P. 52-58.

14. Clements J. Pulmonary surface tension and alveolar stability. // Technical report. CRDLR. U.S. Army Chemical Research and Development Laboratories. 1961. - Vol. 16. - P. 444-450.

15. Hildebran J. Pulmonary surface film stability and composition. // J. of Applied Physiology Respiratory Environmental and Exercise Physiology. - 1979. - Vol. 47(3). - P. 604-611.

16. Meyer K. Bronchoalveolar lavage as a diagnostic tool. // Seminars in Respiratory and Critical Care Medicine. - 2007. - Vol. 28(5). - P. 546-560.

17. Walters E., Gardiner P. Bronchoalveolar lavage as a research tool // Thorax. - 1991. - Vol. 46(9). - P. $613-618$.

18. Horvath I. Exhaled breath condensate contains more than only volatiles. // European Respiratory Journal. 2003. - Vol. 22. - P. 187-188.

19. Kazakov V.N., Sinyachenko O.V., Fainerman V.B., Pison U., Miller R. Dynamic Surface Tensiometry in Medicine. Studies in Interface Science. - 2000, Elsevier.

20. Hinds W.C. Aerosol technology: properties, behavior, and measurement of airborne particles. - 1999, John Wiley \& Sons.

21. Morozov V.N., Mikheev A.Y. A collection system for dry solid residues from exhaled breath for analysis via atomic force microscopy // Journal of Breath Research. - 2017. - Vol. 11. - P. 016006.

22. Pardon G., Ladhani L., Sandstrm N., Ettori M., Lobov G., van der Wijngaart W. Aerosol sampling using an electrostatic precipitator integrated with a microfluidic interface. // Sensors and Actuators B: Chemical. 2015. - Vol. 212. - P. 344-352.

23. Notter $R$. Lung Surfactants: Basic Science and Clinical Applications (Lung Biology in Health and Disease). P.: CRC Press, 2000; 464 p.

24. Bykov A., Loglio G., Miller R., Milyaeva O., Michailov A., Noskov B. Dynamic properties and relaxation processes in surface layer of pulmonary surfactant solutions // Colloids and Surfaces A: Physicochemical and Engineering Aspects. - 2019. - Vol. 573. - P. 14-21.

25. Hohlfeld J. The role of surfactant in asthma // Respiratory Research. - 2001. - Vol. 30. - P. 1201-1204. 
26. Baritussio A. Lung surfactant, asthma, and allergens - A story in evolution. // American Journal of Respiratory and Critical Care Medicine. - 2004. - Vol. 169. - P. 550-551.

27. Wright T., Notter R., Wang Z., Harmsen A., Gigliotti F. Pulmonary inflammation disrupts surfactant function during Pneumocystis carinii pneumonia. // Infection and Immunity. - 2001. - Vol. 69. - P. 758-764.

28. Schwab U., Rohde K.H., Wang Z., Chess P.R., Notter R.H., Russell D.G. Transcriptional responses of Mycobacterium tuberculosis to lung surfactant // Microbial Pathogenesis. - 2009. - Vol. 46. - P. 185-193.

29. Raghavendran K., Willson D., Notter R.N. Surfactant Therapy for Acute Lung Injury and Acute Respiratory Distress Syndrome // Critical Care Clinics. - 2011. - Vol. 27. - P. 525-559.

30. Chroneos Z.C., Midde K., Sever-Chroneos Z., Jagannath C. Pulmonary surfactant and tuberculosis // Tuberculosis. - 2009. - Vol. 89. - P. 10-14.

31. Chimote G., Banerjee R. Lung surfactant dysfunction in tuberculosis: Effect of mycobacterial tubercular lipids on dipalmitoylphosphatidylcholine surface activity // Colloids and Surfaces B: Biointerfaces. - 2005. Vol. 45. - P. 215-223.

32. Hasegawa T., Leblanc R.M. Aggregation properties of mycolic acid molecules in monolayer, 2003.

33. Wang Z., Schwab U., Rhoades E., Chess P.R., Russell D.G., Notter R.H. Peripheral cell wall lipids of mycobacterium tuberculosis are inhibitory to surfactant function // Tuberculosis. - 2008. - Vol. 88. P. $178-186$.

34. Shmyrov A., Mizev I., Mizeva A., Shmyrova A. Electrostatic precipitation of exhaled particles for tensiometric examination of pulmonary surfactant // Journal of Aerosol Science. - 2021. - Vol. 151. - ID 105622.

35. Shmyrov A. [et al.] Capillary wave method: An alternative approach to wave excitation and to wave profile reconstruction //Physics of Fluids. - 2019. - T. 31. - №. 1. - 012101. DOI 10.1063/1.5060666/

36. Шмырова А.И., Мизева И.А., Артамонова П.А. Модификация метода капиллярных волн // Вестник Пермского университета. Сер. Физика. - 2018. - № 3(41). - с. 32-38.

37. Shmyrova A., Shmyrov A., Mizeva I., Mizev A. Registration of high-frequency waves on the surface by the interference methods // EPJ Web of Conferences, - 2019. № 213, 02075. DOI: https://doi.org/10.1051/epjconf/201921302075.

\title{
NOVEL METHODS OF COLLECTING AND ANALYZING LUNG AEROSOL SAMPLES FOR FUNCTIONAL DIAGNOSTICS OF SURFACTANT-DEPENDENT STATES
}

\author{
A.I. Mizev ${ }^{1}$, A.V. Shmyrov ${ }^{1}$, I.A. Mizeva ${ }^{1}$, A.I. Shmyrova ${ }^{1}$, I.M. Pshenichnikova-Peleneva ${ }^{2}$ \\ ${ }^{1}$ Institute of Continuous Media Mechanics UB RAS \\ ${ }^{2}$ Perm State Medical University named after E.A. Wagner
}

\section{For citation:}

Mizev A.I., Shmyrov A.V., Mizeva I.A., Shmyrova A.I., Pshenichnikova-Peleneva I.M. Novel methods of collecting and analyzing lung aerosol samples for functional diagnostics of surfactant-dependent states // Perm Federal Research Center Journal. - 2021. - № 1. - P. 64-72. https://doi.org/10.7242/2658-705X/2021.1.6

The development of effective methods for non-invasive collection of lung aerosol samples and studying the surface activity of the obtained native material is relevant for the diagnosis of inflammatory processes of the lungs. In this work, a method based on the capture of pulmonary fluid droplets contained in the form of an aerosol in the air exhaled by a subject is developed and implemented in hardware - electrostatic aerosol trapping system (ESAT). The exhaled air flow is passed through the corona discharge region, where aerosol droplets receive an electric charge and are then transferred to the water surface by electrostatic forces. The lung surfactant contained in the trapped droplets forms an adsorbed layer, the surface properties of which are then investigated by tensiometric methods.

Testing of the method and optimization of the installation design were carried out on a model aerosol of a weak electrolyte solution, which allowed measuring the capture efficiency through conducting conductometric measurements. A modified capillary wave method was used to study the surface properties of the lung surfactant. The method of collecting exhaled particles and studying the surface properties of the lung surfactant was tested on a group of healthy volunteers. A comparative study of the surface properties of native material collected by the electrostatic 
method from exhaled air and obtained during bronchoalveolar flushing in groups of healthy volunteers and patients with pulmonary tuberculosis was conducted.

Keywords: pulmonary surfactant, surface tension, capillary waves, tuberculosis.

\section{Сведения об авторах}

Мизев Алексей Иванович, доктор физико-математических наук, заведующий лабораторией гидродинамической устойчивости, Институт механики сплошных сред УрО РАН - филиал Пермского федерального исследовательского центра УрО РАН («ИМСС УрО РАН»), 614013, г. Пермь, ул. Академика Королева, 1; e-mail: alex_mizev@icmm.ru Шмыров Андрей Викторович, младший научный сотрудник, «ИМСС УрО РАН»; e-mail: shmyrov@icmm.ru

Мизева Ирина Андреевна, кандидат физико-математических наук, старший научный сотрудник лаборатории физической гидродинамики, «ИМСС УрО РАН»; e-mail: mizeva@icmm.ru Шмырова Анастасия Ивановна, кандидат физико-математических наук, научный сотрудник лаборатории физической гидродинамики, «ИМСС УрО РАН»; e-mail: lutsik @icmm.ru

Пшеничникова-Пеленева Ирина Михайловна, профессор кафедры, доктор медицинских наук, Пермский государственный медицинский университет имени академика Е.А. Вагнера (ПГМУ), 614990, г. Пермь, ул. Петропавловская, 26; e-mail: im_p@ rambler.ru 